\title{
Particle motion in coronary serial stenoses
}

\author{
S. I. Bernad ${ }^{1}$, A. Totorean ${ }^{2}$, E. S. Bernad ${ }^{3} \&$ R. Susan-Resiga ${ }^{2}$ \\ ${ }^{1}$ Romanian Academy, Timisoara Branch, Romania \\ "'Politehnica" University of Timisoara, Romania \\ ${ }^{3}$ University of Medicine and Pharmacy Timisoara, Romania
}

\begin{abstract}
Atherosclerosis creating a constriction can significantly alter the local blood flow dynamics. From a biological aspect, the changes that take place in the flow have a profound effect on the structure and function of the arterial wall and the development of the disease. The purpose of this paper was to non-invasively assess hemodynamic parameters such as wall shear stress, wall pressure and particle depositions with computational fluid dynamics (CFD) in coronary artery serial stenoses. A 3-D model of a serial stenosed RCA was reconstructed based on multislice computerized tomography images. Energy loss associated with such flow expansion after each constriction will be large and consequently the pressure drop will be higher. Pressure drop across the stenoses ST1 and ST3 is lower (4.62 $\mathrm{mmHg}$ and $4.81 \mathrm{mmHg}$ respectively) during the time T2 $=0.79 \mathrm{~s}$, but is significant during the peak systole $\mathrm{T} 1=0.26 \mathrm{~s}$. The maximum WSS in the proximal stenosis ST1 is about $254 \mathrm{~Pa}$, and in the distal stenosis ST3 are $232 \mathrm{~Pa}$. One diameter downstream of the each stenosis, the WSS is low because of the formation of the recirculation zone.

Keywords: blood flow, coronary artery diseases, hemodynamics, pressure drop, wall shear stress.
\end{abstract}

\section{Introduction}

The flow phenomena in the stenotic arteries include asymmetric flow, instabilities and laminar-to-turbulent transition [1-3]. The unsteady flow in a stenotic artery also is characterized by high pressure and wall shear stress (WSS) in the throat and distal to the stenosis [4-7]. 
Significant stenosis produces loss of pressure perfusion and flow to the distal (poststenotic) vascular bed. Energy loss at the stenosis produce a pressure gradient between proximal and distal artery regions [8-11].

The endothelial cell and its interaction with the fluid-induced wall shear stress play a key role in the adaptive response of vessels. The endothelial cell is capable of sensing the level and the direction of the wall shear stress induced by the blood flow, and the biomechanical properties of endothelial cell are partially regulated by the wall shear stress [2, 11-13].

Noninvasive imaging techniques coupled with CFD simulations are important tools for studying the flow field in individual human arterial models, and these numerical models are a convenient platform for evaluating the influence of vessel geometry on pathophysiologically relevant biomechanical properties $[3,5$, $14]$.

\section{Materials and method}

A computer aided design (CAD) model of a human RCA lumen was reconstructed from computed tomographic (CT) scans of a patient (Somatom Sensation 64 Scanner, Siemens Medical Systems, Erlangen, Germany). The patient was a 44-year-old male who had a clinically identified stenosis in his RCA (Figure 1). The RCA geometry was reconstructed based on CT images. Images were then segmented in cross sections perpendicular to the RCA centerline. Figure 2 shows the reconstructed lumen geometry of the RCA viewed from the epicardial. The RCA is modelled to be $52 \mathrm{~mm}$ in length with variable diameters, depending of the stenosis severity. Data corresponding to the reconstructed RCA is presented in Table 1. Good agreement has been demonstrated between coronary artery diameter measurements in the present case and the measurements done by transthoracic echocardiography (TTE) and quantitative coronary angiography (QCA) presented by Kiviniemi et al. [15].

Table 1: $\quad$ RCA stenosis severity (corresponding to the Figure 2).

\begin{tabular}{cccc}
\hline Stenosis & $\begin{array}{c}\text { Stenosis minimal } \\
\text { diameter [mm] }\end{array}$ & $\begin{array}{c}\text { Stenosis length } \\
{[\mathrm{mm}]}\end{array}$ & $\begin{array}{c}\text { Diameter reduction } \\
\text { percentage [\%] }\end{array}$ \\
\hline ST1 & 1.76 & 12 & 54 \\
ST2 & 2.6 & 7 & 28 \\
ST3 & 1.68 & 7 & 53 \\
\hline
\end{tabular}

\subsection{Vascular model}

Figures 2(b) shows the reconstructed lumen geometry of the RCA viewed from the epicardial side. The lumen inlet diameter (at the ostium) of the RCA was $3.8 \mathrm{~mm}$. The percentage diameter reduction for a circular stenosis is $1-\mathrm{d} / \mathrm{D}$ where $\mathrm{d}$ is the diameter of the lumen and $\mathrm{D}$ is the diameter of the unoccluded artery (for the present case diameter of section D3). 


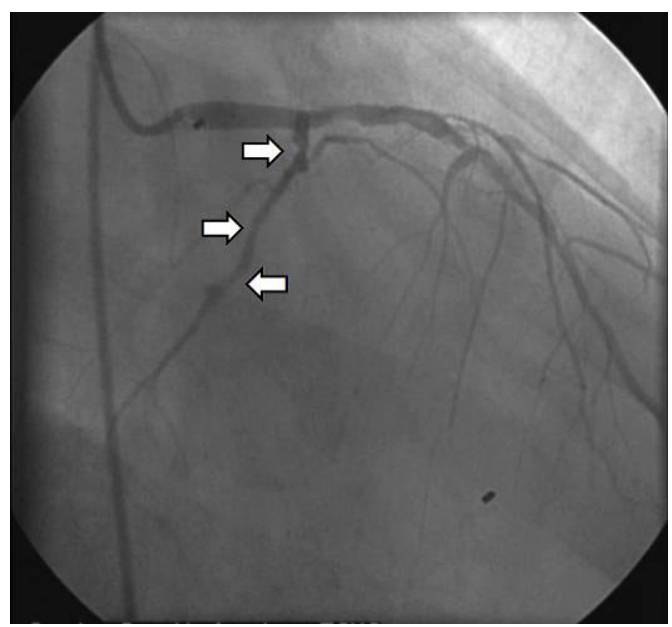

Figure 1: Invasive coronary angiography (CA) image shows the multiple stenosis on the right coronary artery (RCA).

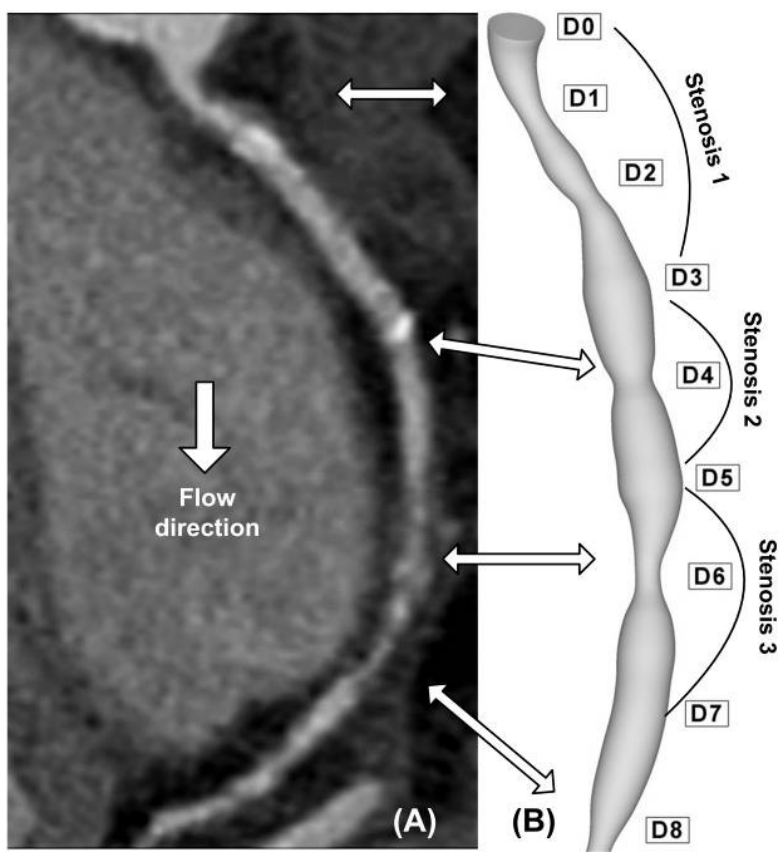

Figure 2: Axial tomographic image which indicates a multiple coronary stenosis at the RCA (arrows); (64-slice CT image). 


\subsection{Numerical model}

Four different meshes with boundary layer mesh were investigated. The number of cells varied between 600,000 and 1,600,000. A high mesh resolution near the walls was needed for accurate values of pressure drop and WSS. Thus the mesh was refined in the near-wall region. A boundary layer consisting of ten rows with a growth factor of 1.15 (ratio between two consecutive layers near the wall) was generated. To model the flow close to the wall, standard wall-function approach was used; then the enhanced wall functions approach has been used to model the near-wall region.

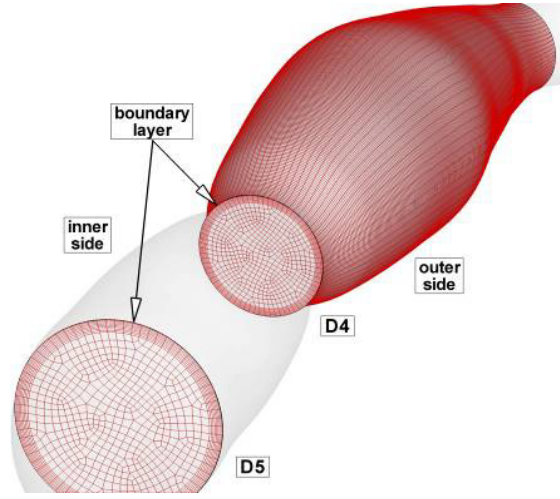

(a)

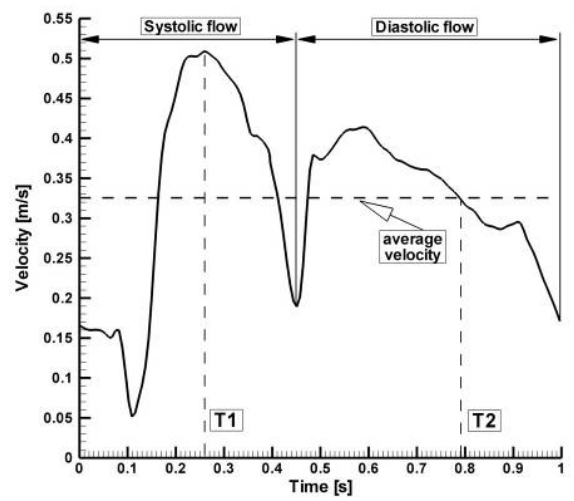

(b)

Figure 3: Patient-specific right coronary artery (RCA) geometry reconstruction (a), computational domain discretization and boundary layer; Physiologically realistic boundary conditions (b), based on flow waveform acquired with an intravascular ultrasound Doppler probe in the RCA [16].

The governing equations are solved iteratively until convergence of all flow variables is achieved. The convergence criterion was set to $10^{-5}$ for the residuals of the continuity equation and of $X, Y$ and $Z$ momentum equations.

Note that recent simulation study of arterial flows presented in the literature $[16,17]$, it shown that about 600,000 grid nodes are required to achieve grid independence in the WSS field. Since our objective is to qualitatively study the unstable flow, the grid containing 1,598,752 cells (Figure 3(a)) can be considered as a good compromise between accuracy and computational cost.

Computational assumptions and boundary conditions are the following: the blood is assumed incompressible having dynamic viscosity $(\mu)$ of $0.00408 \mathrm{~Pa}$ and a density $(\rho)$ of $1050 \mathrm{~kg} / \mathrm{m}^{3}$. The artery walls are considered rigid and impermeable. Johnston et al. [18] compared the effects of different blood viscosity models on the velocity and WSS distributions in the RCA during the cardiac cycle. They concluded that the Newtonian blood model is a reasonably good approximation for transient blood flow simulation. 
The $k-\varepsilon$ model is used to solve the time dependent Navier-Stokes equations for an incompressible blood flow. The numerical simulation is perform using the commercial CFD FLUENT 6.3 package [19], parallelized across eight $3.2 \mathrm{GHz}$ Intel processors of a TYANPSC T-650 Rx (Tyan Computer Corporation, Taiwan, parallel computing machine). The velocity profile at the inlet section is identical to the real coronary ostium velocity profile [16] (Figure 3(b)).

In this model vessel walls are assumed rigid. Zeng et al. [20] have incorporated the effects of physiologically realistic arterial motion into a simulation of blood flow patterns in the RCA. They concluded that arterial motion had little effect on the WSS distribution within the RCA, and that flow in the moving artery followed the instantaneous dynamic geometry quite closely. These results agree with the findings of other groups [21, 22].

\section{Results}

The results of the numerical simulation showed the hemodynamic parameters (pressure distribution and wall shear stress distribution) of the blood flow in the real serial stenosed coronary artery. Our results provide data regarding to the hemodynamic parameters for the blood flow in the stenosed RCA under physiological conditions.

\subsection{Pressure distribution}

The pressure distributions along the axial direction with time for the stenosed RCA are shown in Figure 4. The pressure drop increases with the increase of percentage stenosis. In stenosis ST1 and ST3, the pressure drop is significant during the systolic phase (time T2) $32.84 \mathrm{mmHg}$ respectively $36.78 \mathrm{mmHg}$, while during the diastolic phase at the time T2 the pressure drop across the stenoses ST1 and ST3 is lower (4.62 mmHg and $4.81 \mathrm{mmHg}$ ). For the stenosis ST2, the pressure drop across the stenosis is lower during the peak systolic phase (1.46 mmHg) and practically is negligible during the diastolic phase (0.03mmHg) (Table 2).

Table 2: Hydrodynamic parameters according to the stenotic flow.

\begin{tabular}{|c|c|c|c|c|c|}
\hline Stenosis & Time & $\mathrm{Q}[\mathrm{l} / \mathrm{min}]$ & $\Delta \mathrm{P}[\mathrm{mmHg}]$ & $\mathrm{WSS}_{\max }$ & $\mathrm{WSS}_{\min }$ \\
\hline \multirow{2}{*}{$\mathrm{ST} 1$} & $\mathrm{~T} 1$ & 0.351 & 32.84 & 210 & 10 \\
\cline { 2 - 6 } & $\mathrm{T} 2$ & 0.113 & 4.62 & 45 & 20 \\
\hline \multirow{2}{*}{$\mathrm{ST} 2$} & $\mathrm{~T} 1$ & 0.37 & 1.46 & 110 & 10 \\
\cline { 2 - 6 } & $\mathrm{T} 2$ & 0.118 & 0.03 & 20 & 10 \\
\hline \multirow{2}{*}{ ST3 } & $\mathrm{T} 1$ & 0.35 & 36.78 & 250 & 10 \\
\cline { 2 - 6 } & $\mathrm{T} 2$ & 0.112 & 4.81 & 50 & 10 \\
\hline
\end{tabular}


174 Modelling in Medicine and Biology X

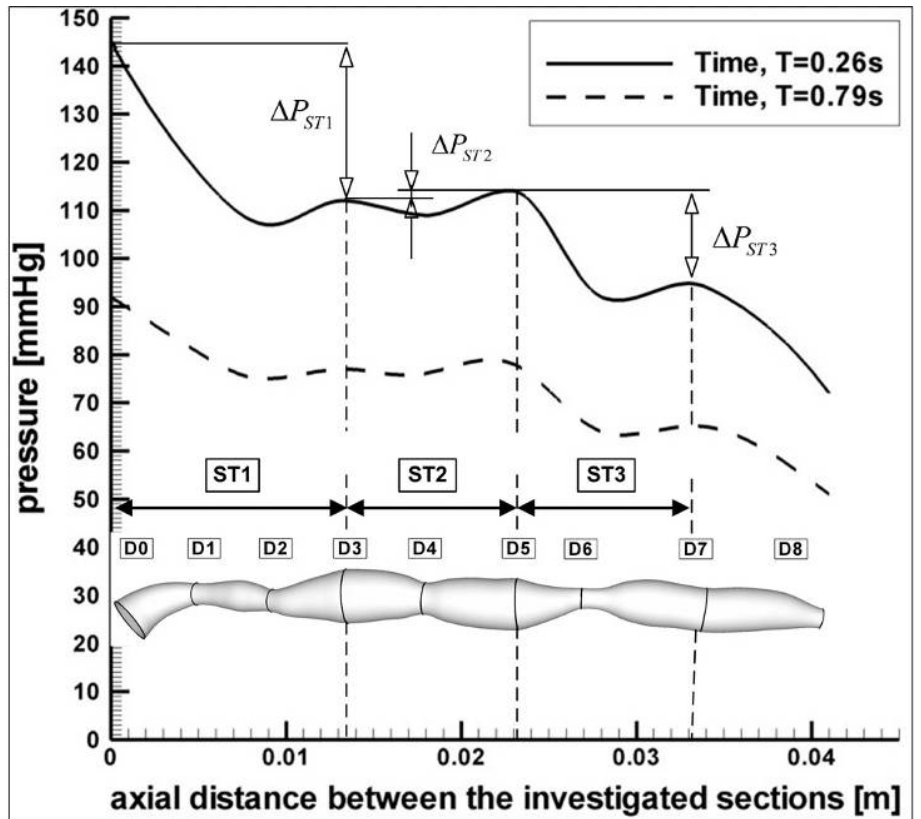

Figure 4: $\quad$ Pressure drop across the investigated serial stenosed RCA.

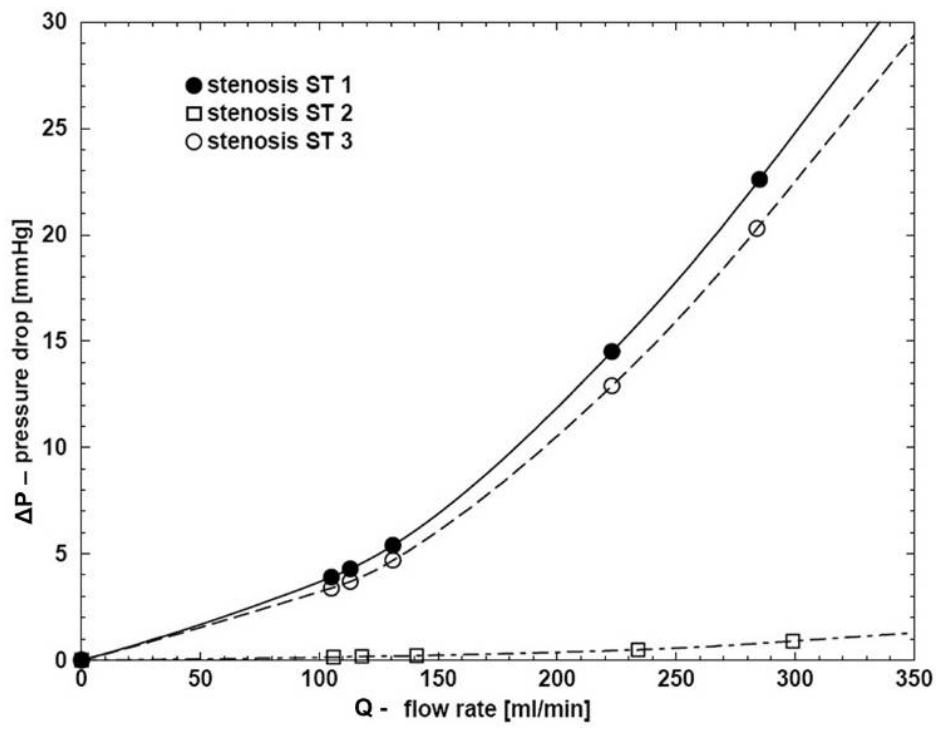

Figure 5: $\quad$ Pressure drop for time step $\mathrm{T} 1$ in the investigated stenosis (T1 correspond to the time steps indicated in Figure 3(b)). 


\subsection{Wall shear stress distribution}

Figure 6 shown the wall shear stress patterns at the peak systole $(T=0.26 \mathrm{~s})$. As presented in in Figure 6 the maximum shear stresses occur in the vicinity of the curvature. Wall shear stress variation during the cardiac cycle is correlated to blood velocity variation. Pressure distribution and wall shear stress are the relevant parameters from the fluid mechanics point of view. According to the Law of Bernoulli as blood velocity increased the pressure is dropped. Energy loss is associated with disturbed flow after each constriction. The greater energy loss induce greater pressure drop associated with large re-circulation zones distal to the each narrowed section.

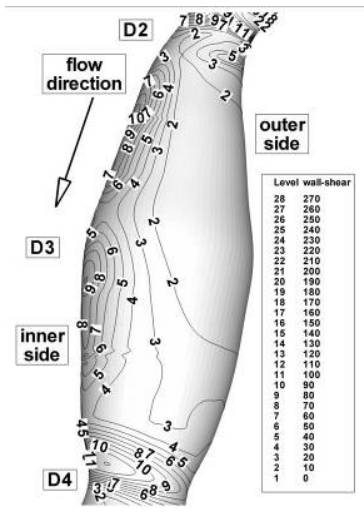

(a)

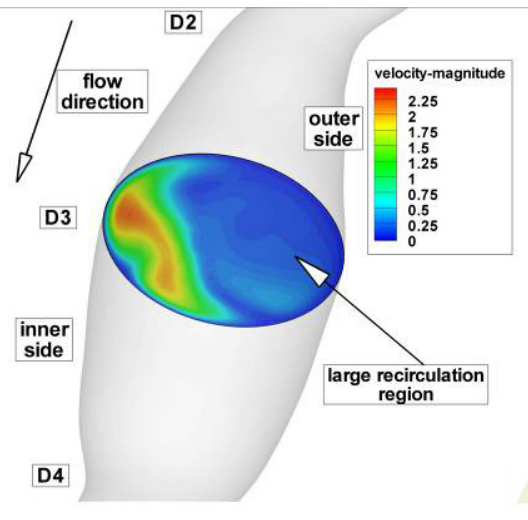

(b)

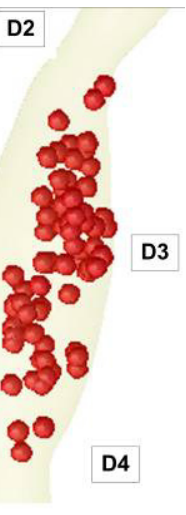

(c)

Figure 6: Disturbed flow indicators at the peak systole T1=0.26 s. (a) temporal WSS magnitude contours around to the cross section D3 (WSS value are in [PA]; (b) cross-sectional transient velocity vector fields; (c) particle deposition patterns.

In Figure 6, the wall shear stress distribution shows a close reflection of the outline of the stenoses. The point where the maximum shear stress occurs lies at the narrowest cross-section D4 for stenosis ST2 and section D6 for stenosis ST3 (Figures 6(a) and 7(a)). There is a rapid increase in wall shear stress upstream of the first stenosis, then after the critical height of stenosis (section D2 and D4), the wall shear stress decreases until separation occurs. The maximum value of wall shear stress is at the critical height of the distal stenosis ST3 (Figure 7(a)). A large separation zone is observed also downstream to the stenosis ST3 (characterized by low WSS region). The peak of wall shear stress is at the critical height of both stenoses, approximately with the same value.

Figure 6 shows wall shear stress (WSS) distributions at the time T1 $=0.26 \mathrm{~s}$, the time where the flow is at a maximum (peak systole). The range of WSS in this figure varies from 7 to $262 \mathrm{~Pa}$. Three intense regions of low WSS appear downstream at the each stenosis (Figures 6 and 7). For each of these regions the 
WSS is approximately $7 \mathrm{~Pa}$. The maximum WSS in the stenosis ST1 is about $254 \mathrm{~Pa}$, rise to $110 \mathrm{~Pa}$ for the stenosis ST2 and $232 \mathrm{~Pa}$ in ST3. One diameter downstream of the each stenosis, the WSS is low because of the formation of the recirculation zone.

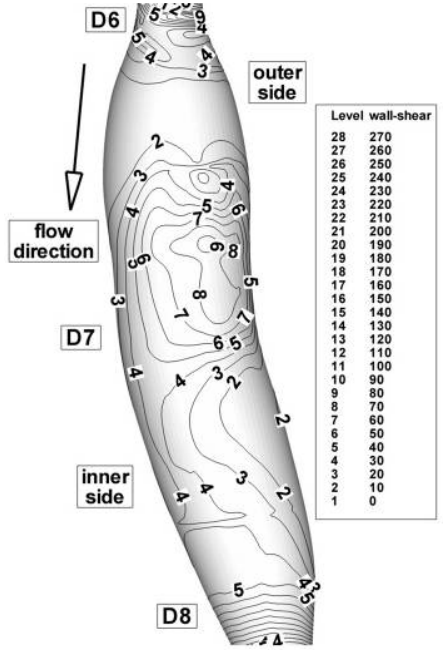

(a)

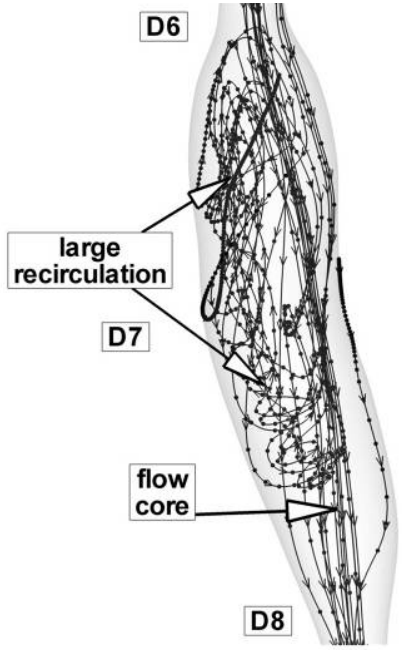

(b)

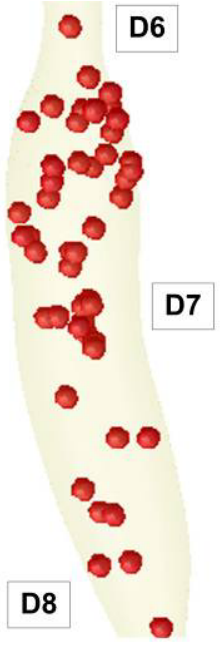

(c)

Figure 7: Disturbed flow indicators sistal to the stenosis ST3 at the peak systole T1=0.26 s. (a) temporal WSS magnitude contours around to the cross section D7 (WSS value are in [PA]; (b) cross-sectional transient velocity vector fields; (c) particle deposition patterns.

Table 3: Minimum/maximum wall shear stress $(\mathrm{Pa})$ in the cross-sections of the stenosed artery.

\begin{tabular}{ccc|cc}
\hline Section & \multicolumn{2}{c|}{ Time T1 $=0.26 \mathrm{~s}$} & \multicolumn{2}{c}{ Time T2=0.79s } \\
\hline & WSS min [Pa] & WSS max [Pa] & WSS min [Pa] & WSS max [Pa] \\
\hline D0 & 7 & 14.00 & 4 & 5.00 \\
D1 & 140 & 168.00 & 88 & 100.00 \\
D2 & 63 & 133.00 & 36 & 124.00 \\
D3 & 7 & 49.00 & 4 & 28.00 \\
D4 & 21 & 91.00 & 24 & 52.00 \\
D5 & 7 & 9.00 & 4 & 8.00 \\
D6 & 154 & 238.00 & 100 & 148.00 \\
D7 & 7 & 56.00 & 4 & 32.00 \\
D8 & 196 & 231.00 & 132 & 140.00 \\
\hline
\end{tabular}




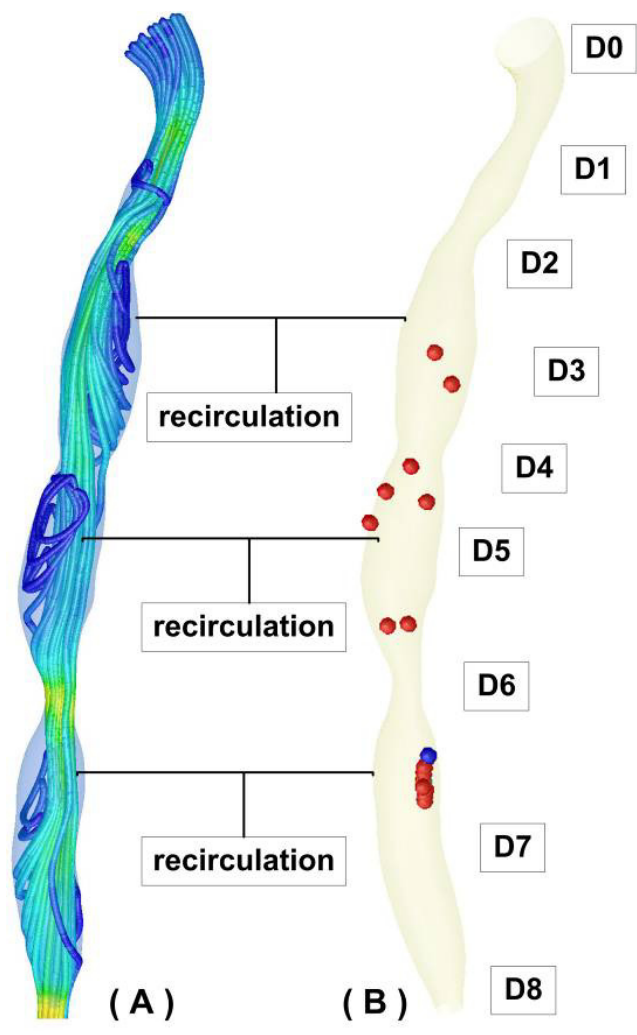

Figure 8: $\quad$ Particles pattline and particles deposition during the investigated time $\mathrm{T} 2=0.79 \mathrm{~s}$.

The particle behaviour shows that the distal region near the stenosis wall is a susceptible place for extended particle entrainment, aggregation, and possibly for particle deposition where particle residence time is prolonged (Figures 6(c), 7(c) and 8). At time level T2 the jet washes a high concentration of particles from the area behind the stenosis. The vortex downstream to each stenosis pushes this elevated particle suspension to the core region of the flow where it is then convected downstream.

Figure 8 represents the paths of fluid particles released in the inlet region at the beginning of the cardiac cycle, and the particle depositions at the time $\mathrm{T} 2=$ 0.79 s. Figures $6-8$ show that the particle residence time and particle deposition increase significantly due to the severe occlusion.

\subsection{Study limitations}

Some limitations of our study should be pointed out. First, the heart movement and the movement of the coronary arteries due to muscle tension cannot be simulated yet, therefore, not included in the present calculations. Second, we 
modelled only the trunk of the RCA by ignoring all of its branches. Third, in this model vessel walls are assumed rigid.

\section{Conclusion}

In patients with multiple stenoses within the same coronary artery, coronary pressure investigation can uniquely determine the separate hemodynamic effects of the individual stenoses in sequence [24]. For clinical practice, pressure recording provides important information, which can help objectively select which of several stenoses is most appropriate for PTCA.

The influence of a distal stenosis on the hemodynamic appearance of a proximal stenosis is generally larger than that of a proximal stenosis on a distal one (Table 2 and 3).

The fluid dynamic interaction of multiple serial stenoses in coronary arteries is complex and cannot be adequately assessed by visual interpretation on the coronary angiogram. Assessment of shear stress, along with percent stenosis, in arterial lesions might prove to be a valuable diagnostic tool to identify patients at risk of developing platelet formation in stenosed arteries. Results in different degrees of stenoses show that severe stenosis caused considerably large pressure drop across the throat. Computer flow models can be utilized in conjunction with human coronary angiography to study flow characteristics for plaques of different sizes and shapes in patients with stable and unstable angina.

\section{References}

[1] J.R Buchanan., C. Kleinstreuer, Simulation of particle-hemodynamics in a partially occluded artery segment with implications to the initiation of microemboli and secondary stenoses, Journal of Biomechanical Engineering, 120, 446-454, 1998.

[2] J.-J. Ciu, D.L. Wang, S. Chien, R. Skalak, S. Usami, Effects of disturbed flow on endothelial cells, Journal of Biomechanical Engineering, 120, 2-8, 1988.

[3] I. Marsahall, S. Zhao, P. Papathanasopoulou, P. Hoskins, X. Yun Xu, MRI and CFD studies of pulsatile flow in healthy and stenosed carotid bifurcation models, Journal of Biomechanics, 37, 679-687, 2004.

[4] N. Sun, R. Torii, N.B. Wood, A.D. Hughes, S. Thom, Y.X. Xu, Computaional modeling of LDL and albumin transport in an in vivo CT image-based human right coronary artery, Journal of Biomechanical Engineering, 131, 1-9, 2009.

[5] T. Frauenfelder, E. Boutsianis, T. Schertler, et al., In-vivo flow simulation in coronary arteries based on computed tomography datasets: feasibility and initial results, Eur Radiol, 17(5), 1291-1300, 2007.

[6] S.I. Bernad, E. Bernad, T. Barbat, V. Albulescu, R. Susan-Resiga, Effects of different types of input waveforms in patient-specific right coronary atherosclerosis hemodynamics analysis, Int. J. of Design \& Nature and Ecodynamics, 5(2), 1-18, 2010. 
[7] S.I. Bernad, E.S. Bernad, Coronary venous bypass graft failure, hemodynamics parameters investigation, Proc. of the IASTED International Conference Biomedical Engineering (BioMed 2012), Innsbruck, Austria, 509-515, 2012.

[8] B. Liu, The influences of stenosis on the downstream flow pattern in curved arteries, Medical Engineering \& Physics, 29, 868-876, 2007.

[9] S.J. Sherwin, H.M. Blackburn, Three-dimensional instabilities and transition of steady and pulsatile axisymmetric stenotic flows, J. Fluid Mech., 533, 297-327, 2005.

[10] S.R. Dodds, The haemodynamics of asymmetric stenoses, Eur J Vasc Endovasc Surg, 24, 332-337, 2002.

[11] D. Zeng, E. Boutsianis, M. Ammann, K. Boomsma, S. Wildermuth, D. Poulikakos, A study of the compliance of a right coronary artery and its impact on wall shear stress, Journal of Biomechanical Engineering, 130, 041014-11, 2008.

[12] G.A. Truskey, K.M. Barber, T.C. Robey, A.O. Lauri, M.P. Combs, Characterization of a sudden expansion flow chamber to study the response of endothelium to flow recirculation, Journal of Biomechanical Engineering, 117, 203-210, 1995.

[13] C.M. Gibson, L. Diaz, K. Kandarpa, et al., Relation of vessel wall shear stress to atherosclerosis progression in human coronary arteries, Arterioscler. Thromb, 13(2), 310-315, 1993.

[14] S. Tateshima, K. Tanishita, F. Vinuela, Hemodynamics and cerebrovascular disease, Surgical Neurology, 70, 447-453, 2008.

[15] T.O. Kiviniemi, M. Saraste, J.W. Koskenvuo, et al., Coronary artery diameter can be assessed reliably with transthoracic echocardiography, Am J Physiol Heart Circ Physiol, 286, H1515-H1520, 2004.

[16] R. Torii, N.B. Wood, N. Hadjiloizou, A.W. Dowsey, A.R. Wright, A.D. Hughes, J. Davies, D.P. Francis, J. Mayet, G-Z. Yang, S.A.McG. Thom, X.X. Yun, Fluid-structure interaction analysis of a patient-specific right coronary artery with physiological velocity and pressure waveforms, Communications in Numerical Methods in Engineering, 25, 565-580, 2009.

[17] V.T. Rayz, S. A. Berger, D. Salomer, Transitional flow in arterial fluid dynamics, Comput. Methods Appl. Mech. Engrg., 196, 3043-3048, 2007.

[18] B.M. Johnston, P.R. Johnston, S. Corney, D. Kilpatrick, Non-Newtonian blood flow in human right coronary arteries: transient simulations, Journal of Biomechanics, 39, 1116-1128, 2005.

[19] FLUENT 6.3 User's Guide, Ansys Fluent Incorporated, 2006.

[20] D. Zeng, Z. Ding, M.H. Friedman, C.R. Ethier, Effects of Cardiac Motion on Right Coronary Artery Hemodynamics, Annals of Biomedical Engineering, 31, 420-429, 2003.

[21] Y. Qiu, J.M. Tarbell, Numerical simulation of pulsatile flow in a compliant curved tube model of a coronary artery. J. Biomech. Eng., 122, 77-85, 2000 . 
[22] A. Santamarina, E. Weydahl, J.M. Siegel Jr., J.E. Moore Jr., Computational analysis of flow in a curved tube model of the coronary arteries: Effects of time-varying curvature, Ann. Biomed. Eng., 26, 944-954, 1998.

[23] R.T. Schoephoerster, F. Gynes, G. Nunez, M. Kapadvanjwala, M.K. Dewanjee, Effects of Local Geometry and Fluid Dynamics on Regional Platelet Deposition on Artificial Surfaces, Arteriosclerosis and Thrombosis, 13(12), 1806-1813, 1993.

[24] Nico H.J. Pijls, De Bruyne Bernard, G. Jan Willem Bech, L. Francesco, R. Guy, J.R.M. Hans, Bonnier and Jacques J. Koolen, Coronary Pressure Measurement to Assess the Hemodynamic Significance of Serial Stenoses Within One Coronary Artery: Validation in Humans, Circulation, 102, 2371-2377, 2000. 